\title{
Retrospective Analysis of Spectrum of Presentation and Treatment Outcome in Extremity Sarcomas: A Single-Centre Experience
}

\author{
Saurabh Bansal, ${ }^{1}$ Kunal Das $\mathbb{D D}^{2}{ }^{2}$ Navneet Jain, ${ }^{3}$ Vipul Nautiyal, ${ }^{1}$ Meenu Gupta, \\ Nadia Shirazi, ${ }^{4}$ Sanjiv Verma, ${ }^{5}$ Mushtaq Ahmad, ${ }^{1}$ and Sunil Saini ${ }^{3}$ \\ ${ }^{1}$ Department of Radiotherapy, Cancer Research Institute, SRHU, Dehradun, Uttarakhand, India \\ ${ }^{2}$ Department of Medical Oncology \& Hematology, Cancer Research Institute, SRHU, Dehradun, Uttarakhand, India \\ ${ }^{3}$ Department of Surgical Oncology, Cancer Research Institute, SRHU, Dehradun, Uttarakhand, India \\ ${ }^{4}$ Department of Pathology, Cancer Research Institute, SRHU, Dehradun, Uttarakhand, India \\ ${ }^{5}$ Department of Medical Oncology, Cancer Research Institute, SRHU, Dehradun, Uttarakhand, India \\ Correspondence should be addressed to Kunal Das; drkunaloncology@gmail.com
}

Received 27 October 2017; Revised 3 January 2018; Accepted 6 March 2018; Published 1 April 2018

Academic Editor: R. Lor Randall

Copyright (c) 2018 Saurabh Bansal et al. This is an open access article distributed under the Creative Commons Attribution License, which permits unrestricted use, distribution, and reproduction in any medium, provided the original work is properly cited.

\begin{abstract}
Introduction. The most common site for soft tissue sarcoma is extremity. As complete surgical resection is possible in majority, outcome of this subset is relatively better. There is paucity of data regarding extremity soft tissue sarcoma (STS) from subHimalayan and hilly geographical regions. Materials and Methods. Retrospective analysis was done for extremity STS visiting the study center over a period of 5 years. Data were collected and analyzed for demography, disease characteristics, treatment modalities, and outcome. Result. Extremity STS constituted $32.8 \%$ of all STS enlisted. Most common subtype noted was pleomorphic STS. Metastatic disease at presentation was noted among 7/43 cases with lung being the most common metastasis site. Wide local excision was done in 37 cases while amputation was required in 5 cases. Adjuvant radiotherapy was given in 27 cases while 18 cases received adjuvant chemotherapy. At median follow-up of 47 months, the overall survival and event-free survival were noted as $47.64 \%$ and $41.49 \%$, respectively. Conclusion. This study depicts single-center experience of extremity STS. The population analyzed was from sub-Himalayan region with significant lost to follow-up. Pooling of data from different centers has been advocated to derive conclusive results.
\end{abstract}

\section{Introduction}

Soft tissue sarcoma (STS) group of tumors are heterogeneous histologically distinct entities. It comprises around $1 \%$ of all malignancies in adult [1]. Extremities STS are the most common anatomical presentation of STS. The possibility of wide local excision and good local control makes it different from intraabdominal and head and neck STS. Surgery remains the mainstay of treatment supplemented by radiation therapy. While adjuvant radiotherapy is recommended for intermediate- and high-grade sarcomas, the role of adjuvant chemotherapy is still debatable. The data regarding STS clinicobiological behavior and outcome is sparse from subHimalayan region and similar high-altitude geographical regions. Even Indian data are sparse and limited to individual center experiences.
The Cancer Research Institute, Dehradun, is the largest and single referral tertiary cancer center in the state of Uttarakhand, India. We present our experience of soft tissue sarcoma occurring primarily in extremities.

\section{Materials and Methods}

A retrospective analysis was done for the patients diagnosed with extremities soft tissue sarcomas from January 2011 to December 2015. Electronic database of the hospital was searched for the patient details (maintained by the Uttarakhand State Council for Science and Technology support) and paper records were retrieved from medical record department. Institutional ethical committee clearance was taken beforehand. The details regarding the demographic profile of patients, anatomical and histological characteristics 
of STS, and treatment modalities and follow-up were collected. Records with insufficient or incomplete information regarding histology or treatment were excluded. Patients enrolled for second opinion were excluded, as no treatment modality was used. The outcome was assessed by electronic record of last visits and telephonic confirmation of survival or demise. Subsets that were not reachable telephonically were tried to contact by post.

Confirmation of diagnosis was done by core needle biopsy or incisional biopsy in all cases. All cases underwent staging workup with computer tomography (CT) of chest and involved limb magnetic resonance imaging. The 2013 WHO Classification for soft tissue sarcoma was used for tumor grouping. Sarcomas lacking characteristic histology, immunohistochemistry, and/or genetic features were categorized as unclassified/undifferentiated sarcoma [2]. Tumor grade was decided based on differentiation and mitotic figures and necrosis according to the Federation Nationale des Centers de Lutte Contre le Cancer (FNCLCC) system. Size, nodal status, and metastasis were noted as per AJCC staging system [3]. Surgical margin status was noted as clear or involved (microscopic or gross residual). A 6-week follow-up for initial two visits followed by 3 monthly visits for next 2 years was advocated to all cases as per institutional protocol. After 2 years of completion of therapy, a 6-month follow-up was planned for each case. Locoregional clinical examination was done at each visit, and annual imaging with CT chest was done. A relapse was confirmed histologically, and local as well as metastatic workup was done to define the pattern of relapse. Treatment-related toxicities requiring admission or intervention were recorded.

2.1. Statistical Analysis. The collected data was analyzed for demographic characteristics, tumor characteristics, and treatment modalities. Statistical analysis was done using SPSS version 17.0 (SPSS Inc., Chicago, IL). Time interval to local recurrence or distant failure was calculated from the date of start of treatment to date of recurrence detection, with censoring at date of death or last contact. Overall survival was calculated from treatment onset to date of death irrespective of the cause of death, with censoring at the date of last contact for the patient alive. Actuarial survival rates were calculated using the Kaplan-Meier method.

\section{Result}

During study period, a total of 250 cases of STS were enlisted. Out of which, 82 were STS of extremities. A cohort of 43 patients was analyzed as 39 cases either visited for opinion with diagnosis done outside or did not opt for surgical treatment in this center. Median age of presentation was 48 years (range 11-75 years), and there was noticeable male dominance (male $:$ female $=28: 15$ ). Commonest histology noted was pleomorphic sarcoma (9/43), followed by synovial (7/43), and liposarcoma (6/43). Lower limb showed preferential site of occurrence in comparison to upper limb. Seven cases presented with metastatic disease with lung being the most common site (5/7). Two cases of each
TABle 1: Tumor characteristics.

\begin{tabular}{lc}
\hline Tumor histology & Numbers \\
Pleomorphic sarcoma & 9 \\
Synovial sarcoma & 7 \\
Spindle cell sarcoma & 5 \\
Liposarcoma & 6 \\
Leiomyosarcoma & 4 \\
Fibrosarcoma & 4 \\
Dermatofibrosarcoma & 3 \\
Malignant peripheral nerve sheath tumor & 2 \\
Unclassified/undifferentiated & 2 \\
Epitheloid sarcoma & 1 \\
Grade & \\
Low grade & 10 \\
Intermediate grade & 4 \\
High grade & 29 \\
Location & \\
Upper limb & 12 \\
Lower limb & 31 \\
Size & \\
T1 (<5 cm) & 9 \\
T2 ( $>5 \mathrm{~cm})$ & 34 \\
Metastasis & \\
M0 & \\
M1 & 36 \\
Stage & 7 \\
Stage I & \\
Stage II & \\
Stage III & \\
Stage IV & \\
\end{tabular}

liposarcoma and synovial sarcoma and one case of leiomyosarcoma, malignant peripheral nerve sheath tumor, and pleomorphic STS each were metastatic at presentation. The median time lag of onset/observation of swelling or pain and diagnosis were 8.5 months. Postoperative grading and TNM was done in all cases. Nine cases were with tumor size $<5 \mathrm{~cm}$ in maximal dimension. Majority (29/43) were high-grade histology while 4 were intermediate and 10 were low-grade sarcoma (Table 1).

Of 36 localized sarcomas, all underwent upfront surgical resection. Wide local excision was a preferred modality and was performed in 33 patients while 3 underwent limb amputation. Of 7 metastatic sarcomas, surgical resection was performed amongst 6 cases, all after first-line chemotherapy while 1 case opted for palliative care (Table 2). Three cases responded well to chemotherapy with disappearance of pulmonary metastasis, and two of them underwent amputation of limb while wide local excision was done in one. One case showed good partial response and underwent lung metastatectomy alone with wide local excision. Two cases showed mild reduction in size and underwent excision in view of local fungating mass over variable period of time. A total of 5 patients underwent amputation. Three were with neurovascular involvement rendering limb salvage difficult while two were with skip bone metastasis at the same limb. Wide local excision was performed in total of 37 cases (33 localized and 4 primary metastatic). Margin positivity was noted among 3 cases, only one underwent re-resection to achieve negative margin. One of them died later with 
TABLE 2: Treatment modalities.

\begin{tabular}{lcc}
\hline Modalities & Nonmetastatic disease & Metastatic disease \\
\hline Surgery & 33 & 4 \\
$\quad$ Wide local excision & 3 & 2 \\
Amputation & 3 & - \\
$\quad$ Margin positivity & & 6 \\
Chemotherapy & 18 & 4 \\
$\quad$ First line & - & 1 \\
$\quad$ Adjuvant & 27 & 3 \\
$\quad$ Palliative & 4 & 4 \\
Radiotherapy & & \\
Relapse of disease/progressive disease & & \\
\hline
\end{tabular}

recurrence of disease while other is alive till analysis. All 3 received adjuvant chemotherapy and radiotherapy to the local site. Postoperative complications noted amongst 3 cases in the form of wound dehiscence in two and partial graft necrosis in one case.

Radiotherapy was administered to 27 cases in adjuvant setting. Indication of radiotherapy involved surgical margin and/or intermediate to high grade of tumor. One case received upfront radiotherapy to primary and metastatic site as palliative care. In metastatic STS cohort, adjuvant radiotherapy was administered to 3 cases at primary site and 2 cases at metastasis site after good response to chemotherapy. The dose of radiotherapy was uniform to all 60-64 Gy in $2 \mathrm{~Gy} /$ day fractionation (external beam radiotherapy using linear accelerator with CT-based three dimensional conformal treatment planning), given in two phases. Phase I included tumor bed, postoperative scar, all drainage sites, and $3-4 \mathrm{~cm}$ margin in longitudinal plane and $1.5-2 \mathrm{~cm}$ margin in transverse plane with a dose of 44-46 Gy. Phase II was delivered for remaining dose on reduced field (tumor bed $+2 \mathrm{~cm}$ margin). Chemotherapy was administered to 6 metastatic cases upfront and 18 as adjuvant (ifosphamideAdriamycin). One patient received low-dose chemotherapy with palliative goal (etoposide-Adriamycin).

18 cases did not turn up for routine follow-up. Telephonic and postal communications resulted in noting outcomes among 5 cases; 13 cases could not be reached and were censored at last point of contact. Among 36 localized sarcomas, recurrence of disease was noted among 4 cases. Recurrence at primary site alone was noted among 3 cases and at lung alone in 1 case. Good locoregional control measures (surgery \pm radiotherapy) resulted in low local recurrence. Of 7 primary metastatic cases, 1 opted for palliative care and died at 4 months. Of remaining 6 cases, three were surviving at the time of analysis, and 3 got recurrences (lung) and died later. With a median follow-up of 47 months (range 7-68 months), the overall survival and event-free survival were noted as $47.64 \%$ and $41.49 \%$, respectively (Figures 1 and 2).

\section{Discussion}

Soft tissue sarcoma of adult encompasses wide histological variants. The most frequent sarcomas noted are liposarcoma, fibrosarcoma, and pleomorphic sarcoma. It can occur throughout the body; however, majority (60\%) occurs in

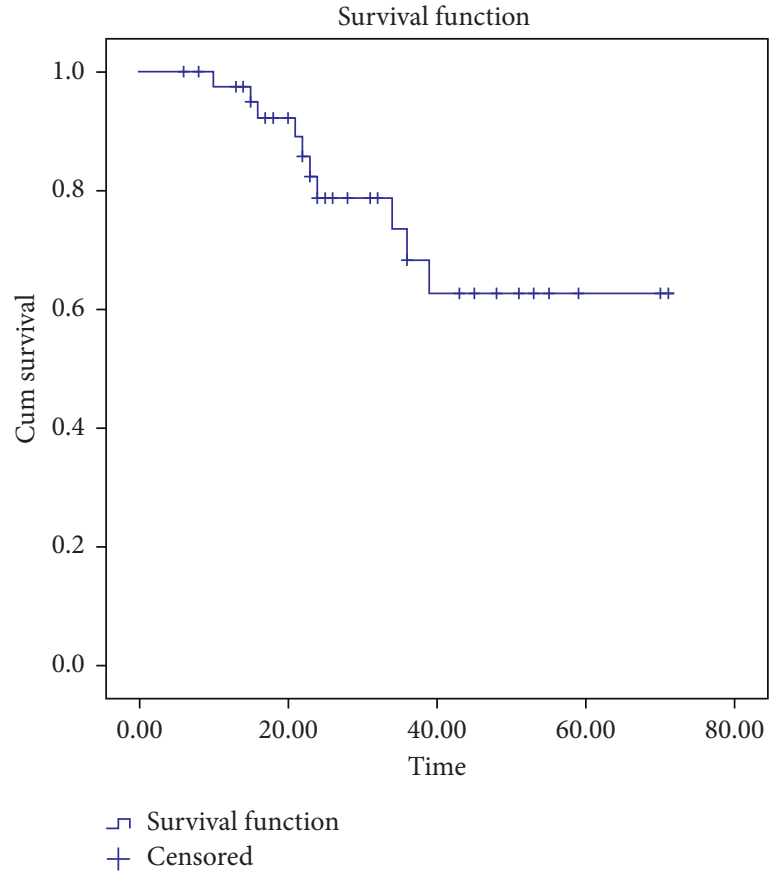

Figure 1: Kaplan-Meier curve showing overall survival (OS).

extremities $[4,5]$. With reason unexplained, lower limb is a more preferred site of occurrence. Probably, large bulk of soft tissue at thigh and leg compartments is the reason. An unusual trend of early age presentation was noted in this study. Male sex has been reported more frequently with STS although this result is not consistent [6-8] The study group also noted $72 \%$ of STS occurrence in lower extremity and male predominance. However, in comparison to all sarcomas reported in center, limb sarcoma constituted only $32 \%$. Cohort noted pleomorphic sarcoma as the most common variant followed by synovial sarcoma. The sample size is small to comment on incidence in general.

Diagnosis of STS of extremities is often delayed. Studies have tried to devise predictive clinical features for occurrence of STS. Swelling exceeding $5 \mathrm{~cm}$, increase in size, pain, and deep location confer more chances of STS in the swelling [9]. Such swelling must be promptly examined by histopathology for STS. Fine-needle aspiration is not a preferred modality, and an excisional biopsy or punch biopsy should be done [10]. In this study, as standard protocol, all diagnosis 


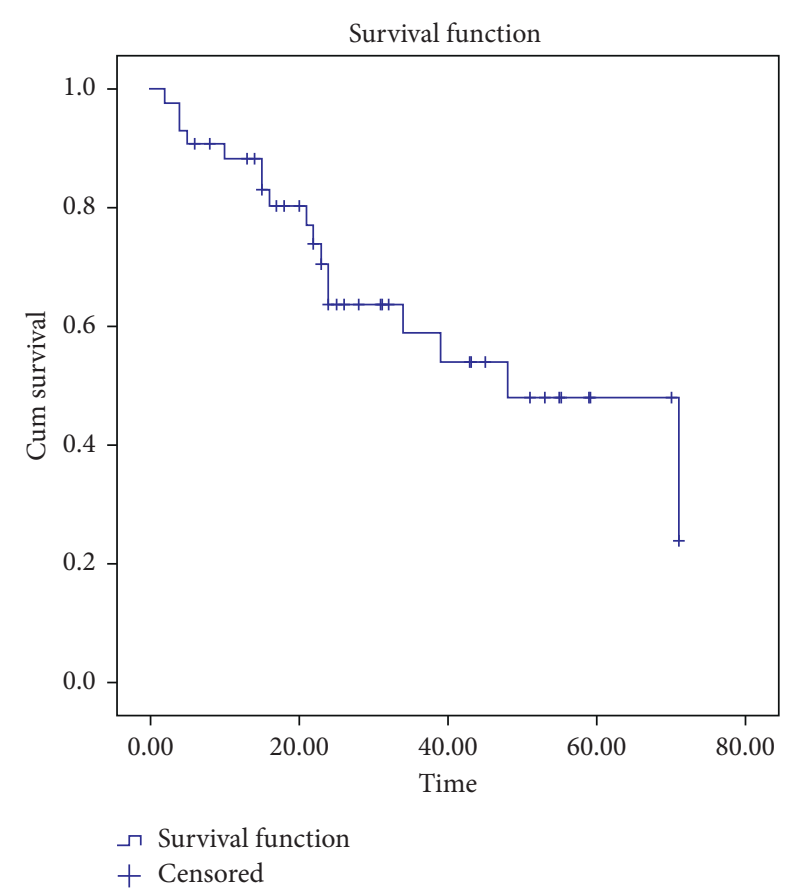

FIgURE 2: Kaplan-Meier curve showing event-free survival (EFS).

was made on histopathology on tissues retrieved from incision or core needle biopsy and confirmed with immunohistochemistry in majority.

Surgical excision of tumor with wide margin, $4-5 \mathrm{~cm}$ to the sides and $1-2 \mathrm{~cm}$ deep to the tumor, is desired. Curative chances are majorly guided by a complete excision. If histopathology result shows narrow or positive margin, a resurgery to achieve a wide margin has clearly shown survival benefit over radiotherapy to margin $[11,12]$. A reresection was advised to cases with positive margin in this cohort; however, it was done in one case only. Re-resection was not technically feasible in one, and resurgery was declined by another. Amputation surgery has a very limited role in current era. In $90 \%$ of extremity STS, limb-sparing excision has been found feasible with local failure rate not exceeding $10 \%$ [13]. An infiltration of major blood vessel or nerve of limb practically makes limb salvage impossible. A plan for reconstruction with plastic surgery team is mandatory for amputation. Study cohort noted five cases of amputation, which happened in either nonsalvageable neurovascular involvement or locally recurrent STS-encasing blood vessel.

Role of adjuvant radiotherapy after a wide local excision is well established in high-grade and intermediate-grade STS. This multimodality achieves about $95 \%$ of local control; however, benefit in terms of overall survival is uncertain $[14,15]$. Radiotherapy alone after a marginal resection is again inferior to reresection. The timing of radiotherapy is also controversial with no superiority of preoperative, or postoperative radiotherapy over another [16-18]. While preoperative radiotherapy makes tumor resectable, a chance of increased wound complications cannot be negated. Trials of perioperative radiotherapy with brachytherapy have been shown comparable to local control with limited radiation effects to nearby structures [19]. A neoadjuvant chemoradiotherapy followed by surgery and postoperative chemotherapy has shown comparable disease control and practically no negative impact of delay in surgery due to intensive neoadjuvant modality [20,21]. Study cohort noted postoperative radiotherapy in 27 cases. It was given to either patient with involved margin or with intermediate-/highgrade feature. Radiotherapy was not given among low-grade tumors that underwent wide local excision, and margins were negative. Chemotherapy has been noted to have limited role except rhabdomyosarcoma and Ewing's group of sarcoma. Various trials have noted conflicting benefit of neoadjuvant/adjuvant chemotherapy with no benefit in overall survival $[22,23]$. For locally advanced STS, reports of regional hyperthermia in combination of chemotherapy have shown better local control and outcome as well. Similarly, option of isolated limb perfusion of affected extremity with chemotherapeutic agents has been reported for primary inoperable limb sarcomas [24-26].

Extremities sarcoma has been noted to have better outcome, and the most likely explanation is complete surgical excision. Various studies have noted survival at 5 years as 65-75\% with local recurrence rate of about 10\% [27-29]. SEER data showed 3-year overall survival of 73\% if radiotherapy was administered in adjuvant setting in high-risk STS [30]. Present study noted inferior overall and event-free survival. Censoring of cases at last follow-up and large number of lost to follow-up might be affecting the actual survival result. As this study analyzed population of hilly terrain, lost to follow-up was significant. In this study, telephonic and postal communication was used to ensure proper follow-up; however, a robust use of telemedicine and messaging by social networking would have been more useful. Study noted large number of high-risk STS which might have contributed to inferior outcome as well. This study presents the experience of extremity STS from a tertiary referral cancer center at sub-Himalayan region. The number of cases is small to draw any definitive conclusion regarding treatment efficacy or environmental effect on outcome. There is a need of pooling data from Himalayan/sub-Himalayan region to delineate effects of this geographical factor.

\section{Disclosure}

Earlier version of part of this work was presented as an abstract at the 2nd Indian Cancer Congress 08-12 November, 2017, Bengaluru, and published as an abstract in Journal of Cancer Research \& Therapeutics, 2017 supplement, vol. 13, pS369, 1/4p.

\section{Conflicts of Interest}

The authors declare that they have no conflicts of interest regarding this study.

\section{Acknowledgments}

The authors duly acknowledge the Uttarakhand State Council for Science and Technology (U-COST) for assistance in data management and compilation. 


\section{References}

[1] A. Jemal, R. Siegel, E. Ward et al., "Cancer statistics," CA: A Cancer Journal for Clinicians, vol. 58, no. 2, pp. 71-96, 2008.

[2] L. A. Doyle, "Sarcoma classification: an update based on the 2013 World Health Organization classification of tumors of soft tissue and bone," Cancer, vol. 120, no. 12, pp. 1763-1774, 2014.

[3] American Joint Committee on Cancer, AJCC Cancer Staging Manual, Springer, New York, NY, USA, 6th edition, 2002.

[4] G. Lahat, D. Tuvin, C. Wei et al., "New perspectives for staging and prognosis in soft tissue sarcomas," Annals of Surgical Oncology, vol. 15, no. 10, pp. 2739-2748, 2008.

[5] N. F. Gilbert, C. P. Cannon, P. P. Lin, and V. O. Lewis, "Softtissue sarcoma," Journal of the American Academy of Orthopaedic Surgeons, vol. 17, no. 1, pp. 40-47, 2009.

[6] H. H. Storm, "Cancers of the soft tissues," Cancer Surveys, vol. 19-20, pp. 197-217, 1994.

[7] J. R. Toro, L. B. Travis, H. J. Wu, K. Zhu, C. D. Fletcher, and S. S. Devesa, "Incidence patterns of soft tissue sarcomas, regardless of primary site, in the surveillance, epidemiology and end results program, 1978-2001: an analysis of 26,758 cases," International Journal of Cancer, vol. 119, no. 12, pp. 2922-2930, 2006.

[8] C. Wibmer, A. Leithner, N. Zielonke, M. Sperl, and R. Windhager, "Increasing incidence rates of soft tissue sarcomas? A population-based epidemiologic study and literature review," Annals of Oncology, vol. 21, no. 5, pp. 1106-1111, 2010.

[9] C. J. Johnson, P. B. Pynsent, and R. J. Grimer, "Clinical features of soft tissue sarcomas," Annals of the Royal College of Surgeons of England, vol. 83, no. 3, pp. 203-205, 2001.

[10] H. A. Domanski, "Fine-needle aspiration cytology of soft tissue lesions: diagnostic challenges," Diagnostic Cytopathology, vol. 35, no. 12, pp. 768-773, 2007.

[11] J. C. Gutierrez, E. A. Perez, F. L. Moffat, A. S. Livingstone, D. Franceschi, and L. G. Koniaris, "Should soft tissue sarcomas be treated at high-volume centers? An analysis of 4205 patients," Annals of Surgery, vol. 245, no. 6, pp. 952-958, 2007.

[12] M. W. Manoso, D. A. Frassica, E. G. Deune, and F. J. Frassica, "Outcomes of re-excision after unplanned excisions of softtissue sarcomas," Journal of Surgical Oncology, vol. 91, no. 3, pp. 153-158, 2005.

[13] V. K. Sondak, "Sarcomas of bone and soft tissue," in Surgery: Scientific Principles and Practice, L. J. Greenfield, M. W. Mulholland, K. T. Oldham, G. B. Zelenock, and K. D. Lillemoe, Eds., pp. 2258-2280, Lippincott Williams \& Wilkins, Philadelphia, PA, USA, 3rd edition, 2000.

[14] H. Strander, I. Turesson, and E. Cavallin-Stahl, "A systematic overview of radiation therapy effects in soft tissue sarcomas," Acta Oncologica, vol. 42, no. 5-6, pp. 516-531, 2003.

[15] T. F. DeLaney, L. Kepka, S. I. Goldberg et al., "Radiation therapy for control of soft-tissue sarcomas resected with positive margins," International Journal of Radiation Oncology, Biology, Physics, vol. 67, no. 5, pp. 1460-1469, 2007.

[16] P. Hohenberger and W. M. Wysocki, "Neoadjuvant treatment of locally advanced soft tissue sarcoma of the limbs: which treatment to choose?," The Oncologist, vol. 13, no. 2, pp. 175-178, 2008.

[17] B. O’Sullivan, A. M. Davis, R. Turcotte et al., "Preoperative versus post-operative radiotherapy in soft-tissue sarcoma of the limbs: a randomized trial," The Lancet, vol. 359, no. 9325, pp. 2235-2241, 2002.

[18] A. S. Lichter and T. S. Lawrence, "Recent advances in radiation oncology," New England Journal of Medicine, vol. 332, no. 6, pp. 371-379, 1995.
[19] S. Laskar, G. Bahl, A. Puri et al., "Perioperative interstitial brachytherapy for soft tissue sarcomas: prognostic factors and long-term results of 155 patients," Annals of Surgical Oncology, vol. 14, no. 2, pp. 560-567, 2007.

[20] P. Anderson, D. Aguilera, M. Pearson et al., "Outpatient chemotherapy plus radiotherapy in sarcomas: improving cancer control with radiosensitizing agents," Cancer Control, vol. 15 , no. 1 , pp. $38-46,2008$.

[21] T. F. DeLaney, I. J. Spiro, H. D. Suit et al., "Neoadjuvant chemotherapy and radiotherapy for large extremity soft-tissue sarcomas," International Journal of Radiation Oncology, Biology, Physics, vol. 56, no. 4, pp. 1117-1127, 2003.

[22] Sarcoma Meta-analysis Collaboration (SMAC), "Adjuvant chemotherapy for localized resectable soft tissue sarcoma in adults," Cochrane Database of Systematic Reviews, no. 4, p. CD001419, 2000.

[23] N. Pervaiz, N. Colterjohn, F. Farrokhyar, R. Tozer, A. Figueredo, and M. Ghert, "A systematic meta-analysis of randomized controlled trials of adjuvant chemotherapy for localized resectable soft-tissue sarcoma," Cancer, vol. 113, no. 3, pp. 573-581, 2008.

[24] R. D. Issels, "Regional hyperthermia as targeted therapy in the management of high-risk soft-tissue sarcoma," European Oncology \& Haematology, vol. 1, no. 2, pp. 119-121, 2007.

[25] R. D. Issels, S. Abdel-Rahman, C. M. Wendtner et al., "Neoadjuvant chemotherapy combined with regional hyperthermia (RHT) for locally advanced primary or recurrent high-risk soft-tissue sarcomas (HR-STS) of adults: long-term results of a phase II study," European Journal of Cancer, vol. 37, no. 13, pp. 1599-1608, 2001.

[26] D. Andreou, M. Werner, D. Pink et al., "Histological response assessment following neoadjuvant isolated limb perfusion in patients with primary, localized, high-grade soft tissue sarcoma," International Journal of Hyperthermia, vol. 32, no. 2, pp. 159-164, 2016.

[27] B. A. Morrison, "Soft tissue sarcomas of the extremities," Baylor University Medical Center Proceedings, vol. 16, no. 3, pp. 285-290, 2003.

[28] P. W. Pisters, D. H. Leung, J. Woodruff, W. Shi, and M. F. Brennan, "Analysis of prognostic factors in 1,041 patients with localized soft tissue sarcomas of the extremities," Journal of Clinical Oncology, vol. 14, no. 5, pp. 1679-1689, 1996.

[29] J. J. Lewis, D. Leung, E. S. Casper, J. Woodruff, S. I. Hajdu, and M. F. Brennan, "Multifactorial analysis of long-term followup (more than 5 years) of primary extremity sarcoma," Archives of Surgery, vol. 134, no. 2, pp. 190-194, 1999.

[30] M. Koshy, S. E. Rich, and M. M. Mohiuddin, "Improved survival with radiation therapy in high-grade soft tissue sarcomas of the extremities: a SEER analysis," International Journal of Radiation Oncology, Biology, Physics, vol. 77, no. 1, pp. 203-209, 2010. 


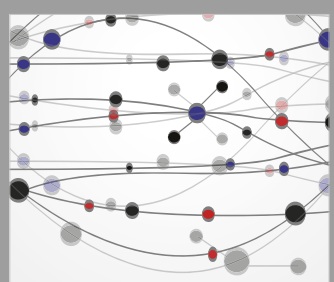

The Scientific World Journal
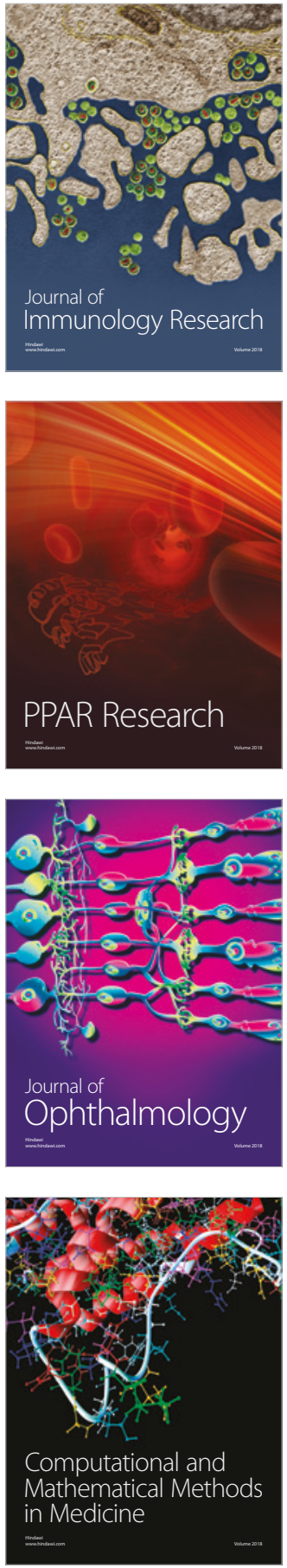

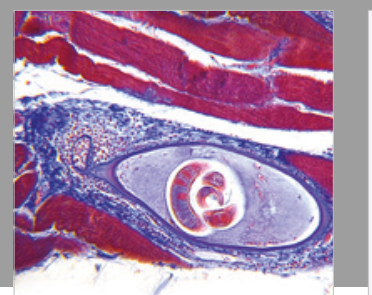

Gastroenterology Research and Practice

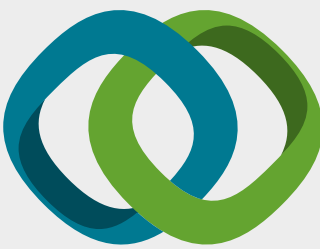

\section{Hindawi}

Submit your manuscripts at

www.hindawi.com
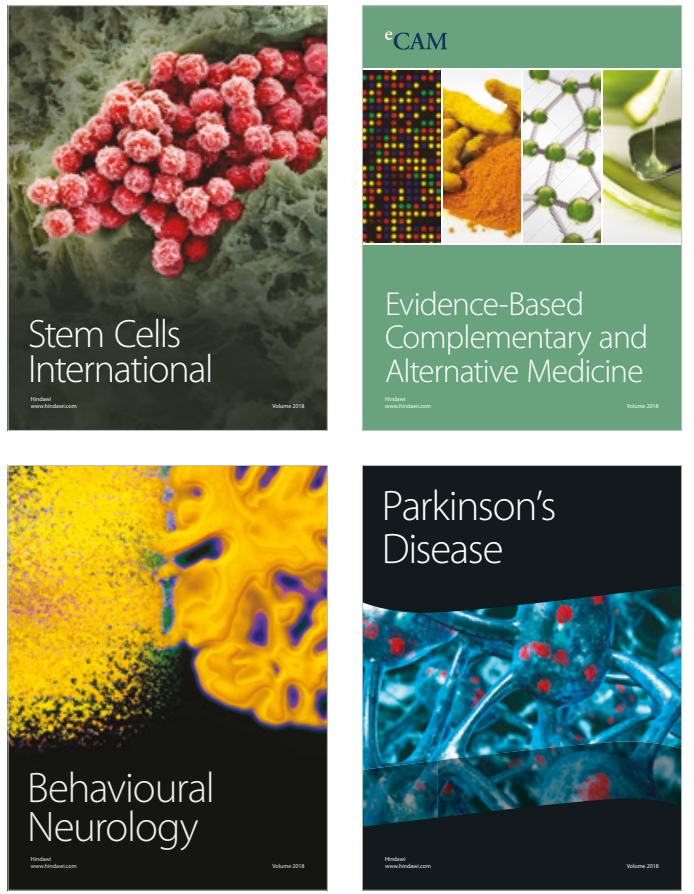

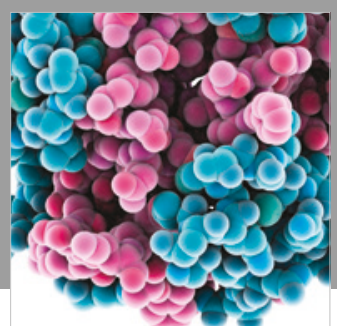

ournal of

Diabetes Research

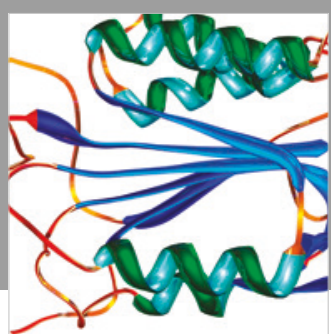

Disease Markers
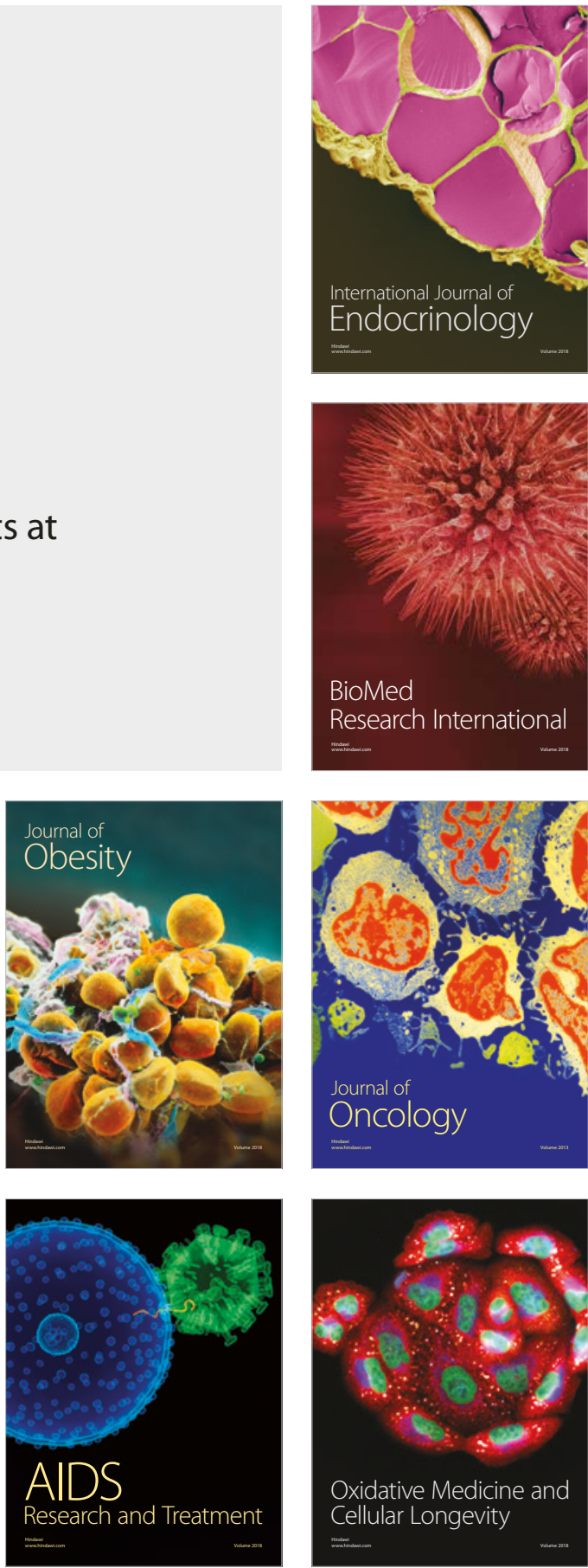